\title{
EXPERIMENTAL VERIFICATION OF CALCULATED LATTICE RELAXATIONS AROUND IMPURITIES IN CdTe
}

\author{
H.-E. Mahnke ${ }^{1 *}$, H. Haas ${ }^{1}$, V. Koteski ${ }^{1,2}$, N. Novakovic ${ }^{1,2}$, P. Fochuk ${ }^{3}$, and O. Panchuk ${ }^{3}$ \\ ${ }^{1}$ HMI Berlin GmbH, Bereich Strukturforschung, D-14109 Berlin, Germany \\ ${ }^{2}$ VINČA, POB 522, 11001 Belgrade, Serbia and Montenegro \\ ${ }^{3}$ University of Chernivtsi, 274012 Chernivtsi, Ukraine
}

\begin{abstract}
We have measured the lattice distortion around As (acceptor) and $\mathrm{Br}$ (donor) in CdTe with fluorescence detected X-ray absorption spectroscopy. We could experimentally verify the lattice relaxation with a bond length reduction of $8 \%$ around the As atom as inferred indirectly from $a b$ initio calculations of the electric field gradient performed with the WIEN97 package in comparison with the measured value in a Perturbed Angular Correlation experiment as recently reported. We have complemented our own calculations of relaxation with WIEN97 with calculations using the FHI96md pseudo-potential program which allows the use of larger super-cell sizes. Encouraged by the good agreement between experiment and model calculation for As in CdTe as well as similarly for the isovalent Se in CdTe, we extended our investigation to $\mathrm{Br}$ in $\mathrm{CdTe}$, where the electric field gradient has also been measured, and could not only verify the derived lattice expansion around $\mathrm{Br}$ with our EXAFS analysis but additionally observe fractions of $\mathrm{Br}$ in the $\mathrm{A}$-center as well as in a DX-center configuration.
\end{abstract}

Keywords: Local structure, lattice relaxation, dopants in CdTe, fluorescence detected X-ray absorption, calculations with DFT theories with LAPW and pseudo-potential methods PACS codes: 61.72.-y , 61.72.Vv, 61.10.Ht , 71.15.Ap , 71.15.Mb , 71.55.Gs 


\section{Introduction}

The electrical and optical properties of semiconductors are determined by the local environment of impurities. Local strain fields around the impurity atom are decisive for the stability of the defect both structurally and functionally as dopant. In the case of As and $\mathrm{Br}$ in CdTe acting as acceptor and donor, resp., when incorporated substitionally on the Te sublattice, information on lattice relaxation around the impurity atom has already been obtained by hyperfine interaction studies. Making use of the donor-acceptor-pair formation between In-As and $\mathrm{Ag}-\mathrm{Br}$ the electric field gradient (EFG) was determined for the non-cubic configuration with the resulting pairs of ${ }^{111} \mathrm{Cd}-\mathrm{As}[1]$ and ${ }^{111} \mathrm{Cd}-\mathrm{Br}$ [2] after the decay of ${ }^{111} \mathrm{In}$ or ${ }^{111} \mathrm{Ag}$, resp., employing the perturbed angular correlation (PAC) technique. In $a b$ initio calculations using density functional theory (DFT) as implemented in the WIEN97 program the EFGs were reproduced when the lattice distances around the impurity atom were first allowed to relax to minimize the forces within the super-cell [1,3]. Since the EFG has a high sensitivity to the actual size of atomic relaxations around the probing atom, it is most desirable to experimentally strengthen this approach by determining the bond lengths around the impurity atom directly, which can be achieved with fluorescence detected X-ray Absorption Fine Structure (XAFS with X-ray Absorption Near-Edge Spectroscopy XANES and Extended X-ray Absorption Fine Structure EXAFS).

The challenge in such experiments lies in the necessary compromise between a concentration high enough for a XAFS detection, but low enough to avoid compensation and clustering of the dopants in the semiconductors. Since a site sensitive XAFS detection has not yet been successfully developed for these systems, one has to be sure that the signal from other possible configurations does not obscure the signal from the assumed purely substitutional one. While for As in CdTe, other conflicting configurations might be various cluster configurations, the most likely alternative configurations for $\mathrm{Br}$ in $\mathrm{CdTe}$ are the well-known A-center [4] or various forms of DX-centers [5]. 


\section{Experiment}

\subsection{Experimental details.}

While the incorporation of dopants like $\mathrm{Br}$ is accomplished in crystal growing from the melt, the incorporation of arsenic as the acceptor dopant in CdTe was done by ion implantation with proper treatment. At the tandetron of FZ Rossendorf, a total dose of $7 \times 10^{15} \mathrm{~cm}^{-2}$-As atoms was implanted almost uniformly distributed up to a depth of $3 \mu \mathrm{m}$. The samples, originally from Crystec $\mathrm{GmbH}^{1}$, were thermally treated before and after the implantation following the approaches [6] in which As was predominantly incorporated as acceptor at the Te site as checked by photoluminescence (PL) measurements. To reduce background in the fluorescence detected EXAFS from undoped sample material, the crystals were thinned from the back to a total thickness of about $30 \mu \mathrm{m}$. In the case of $\mathrm{Br}$ in CdTe, we used powdered samples, mixed with graphite and polyethylene, and pressed into pellets. Concerning the $\mathrm{Br}$ concentration we can only estimate from the concentration in the melt of $5 \times 10^{19} \mathrm{~cm}^{-3}$ that the final $\mathrm{Br}$ concentration is in the range of a few $10^{18} \mathrm{~cm}^{-3}$.

To determine the local structure around the impurity elements we have measured the K-edge absorption at the X1-beamline of HASYLAB at DESY. In the case of As we measured the absorption in the fluorescence mode with the 5-segment Ge-detector, the fluorescence being detected in line with the polarization vector of the incoming synchrotron radiation. To reduce the influence from elastic and inelastic scattering of the incoming radiation, critical absorption was employed using a foil made out of Ge powder mixed with polyethylene. In the case of $\mathrm{Br}$, a 7-segment Ge-detector could be used with an improved resolution yielding a better sensitivity for lower concentrations. For the measurements, the samples were mounted on a He gas flow cryostat, keeping the temperature at either approximately 18 or $300 \mathrm{~K}$.

\footnotetext{
${ }^{1}$ Crystec GmbH, D-12555 Berlin
} 
The analysis of the observed absorption spectra was done following the standard FEFF procedure [7,8] including the background treatment with AUTOBK [9].

\subsection{Theoretical details.}

Complementing our experimental work we have performed $a b$ initio calculations based on the density functional theory (DFT) with the WIEN97 package [10] which uses the linearized augmented plane wave (LAPW) method, confirming and extending the calculation by Lany et

al. [1], and with the FHI96md program [11] which uses first-principles pseudo-potentials (PP) and a plane-wave basis set. The latter was used to investigate the size dependence of the super-cells constructed around one substitutional As atom in CdTe. The calculations yielded good agreement with our EXAFS experiment (see Table1) so that the determined relaxations can be taken as a solid basis for further interpretations of derived parameters such as hyperfine interaction parameters of defect complexes (for further details see $[1,12]$ ).

\section{Results and discussion}

In the case of As in CdTe our EXAFS result yielded a strong inward relaxation of $8 \%$ in perfect agreement with the values calculated using the LAPW method which has been applied in the EFG calculation as well as with the pseudo-potential method with cell-sizes up to 216 atoms. For comparison we have also determined the relaxation around Se, elemental neighbor to As and isovalent to the anion Te, in a mixed crystal $\mathrm{CdTe}_{1-\mathrm{x}} \mathrm{Se}_{\mathrm{x}}$ with a concentration of $\mathrm{x}=0.04$ which comes close to the super-cell size of 32 atoms. Again we find perfect agreement between experiment and model calculation with an inward relaxation of $6 \%$. The results are summarized in Tab.1, more details can be found in ref. [13].

The case of $\mathrm{Br}$ in CdTe already provides the opportunity for a more sophisticated analysis of a more complex doping situation. A first EXAFS analysis yields a bond length which is in fair accordance with the bond length derived in the WIEN calculation in reproducing the measured EFG at Cd with one Br neighbor in the Te nearest neighbor shell (see Tab.1) (the 
preliminary result was already presented at the E-MRS Spring Meeting 2004 [14]). However, compared to the perfect agreement obtained for the cases As and Se (within much less than $1 \%$ ), the analysis seems to be questionable. We have therefore analyzed the absorption signal on $\mathrm{Br}$, both in the XANES region and in the EXAFS region, more carefully. Indeed, the larger width of the first shell signal (in R-space) compared to As indicates the possibility of a superposition of different configurations. Since our $\mathrm{Br}$ concentration is close or even above the maximum for an uncompensated doping concentration, we included the A-center configuration known as a possible compensating configuration in our analysis. In the XANES region, the model absorption spectra are very similar for both, the substitutional $\mathrm{Br}$ and the Acenter $\mathrm{Br}$, resp., however it would be very different for a differently charged $\mathrm{Br}$, for which an illustration was presented already in ref. [14]. There is a clear trend that the XANES spectrum corresponds mainly to positively charged $\mathrm{Br}$ configurations.

In the EXAFS region, including a sizeable fraction of $\mathrm{Br}$ in the A-center configuration significantly improves the fit, however with the distances fixed. A fully satisfactory fit is obtained by including a DX-center configuration as a third contribution of the same size as the substitutional fraction (Fig.1), again however with fixed distances. As distances we took the calculated values $\mathrm{R}_{\mathrm{nn}}=2.90 \AA$ for substitutional $\mathrm{BrTe}, 2.80 \AA$ for the A-center $\mathrm{Br}$, and for the DX-center following ref. [5] 2.60§ for Br-Cd (3-fold coordinated) and $3.10 \AA$ for the more relaxed $\mathrm{Br}-\mathrm{Cd}$ distance. The next-nearest neighbor shell was included with a fixed value taken from the pure system. The occurrence of various configurations which we experience here may also explain the relatively small fraction with which the PAC experiment detected the ${ }^{111} \mathrm{Ag}-\mathrm{Br}$ pair [2].

In summary, in both cases of dopants, the acceptor As as well as the donor $\mathrm{Br}$ in CdTe, for which the determination of the EFG on the neighboring $\mathrm{Cd}$ atom has pointed towards a sizeable change in atomic distances, these distances have now been measured with EXAFS. Both, the large decrease of $8 \%$ for As in CdTe and the remarkable increase of more than $3 \%$ 
for $\mathrm{Br}$ in $\mathrm{CdTe}$ were found in perfect agreement with the WIEN calculation which reproduced the observed EFGs. In the case of As in CdTe, we additionally tested the theoretical approach by using the pseudo-potential program FHI96md which allowed to use a larger super-cell size finding no significant differences. In the case of $\mathrm{Br}$ in CdTe, the absorption spectroscopy revealed the contribution of other configurations to Br substitutionally incorporated which we tentatively identify as the expected A-center and a DX-center.

Acknowledgement: The authors are grateful to the HASYLAB staff at DESY, in particular to N. Haack, E. Welter, and J. Wienold. We thank E. Holub-Krappe for her help in the early phase and H. Rossner for his advice on analysis and fitting of the data and for P. Szimkowiak for his help in sample preparation. Help in sample preparation is also acknowledged for the As implantation by M. Friedrich (FZ Rossendorf), and crystal thinning and PL tests by S. Lany, F. Wagner and H. Wolf (University Saarbrücken). We further thank J. Bollmann and J. Weber from the TU Dresden, S. Lany and T.Wichert from the U Saarbrücken, and B.Cekic from VINČA.

\section{Table 1}

Comparison of experimentally determined nearest neighbor and next-nearest neighbor distances around $\mathrm{As}$, Se and $\mathrm{Br}$ impurity atoms in $\mathrm{CdTe}$ with calculated values obtained with LAPW (super-cell size 32 atoms) and with PP methods (super-cell size up to 216 atoms). The distances in pure CdTe are derived from the standard lattice parameter. (All values are given in units of $0.1 \mathrm{~nm}(=1 \AA))$.

\begin{tabular}{|c|c|c|c|c|c|c|}
\hline \multirow{3}{*}{$\begin{array}{l}\text { System } \\
\text { CdTe }\end{array}$} & \multirow{3}{*}{$R_{n n}(\exp )$} & \multicolumn{2}{|c|}{$\mathbf{R}_{\mathrm{nn}}$ (theo) } & \multirow{2}{*}{$R_{n n n}(\exp )$} & \multicolumn{2}{|c|}{$\mathbf{R}_{\mathrm{nnn}}$ (theo) } \\
\hline & & \multicolumn{2}{|c|}{ LAPW $\quad$ PP } & & \multicolumn{2}{|c|}{ LAPW $\quad$ PF } \\
\hline & & & & 4.583 & & \\
\hline As in CdTe & $2.58(2)$ & $2.57^{\#}$ & 2.57 & $4.50(6)$ & $4.52^{\#}$ & 4.55 \\
\hline Se in CdTe & $2.67(1)$ & 2.66 & & $4.53(1)$ & 4.55 & \\
\hline $\mathrm{Br}$ in $\mathrm{CdTe}$ & $2.83(5)^{*}$ & 2.90 & & $4.54(7)^{*}$ & 4.57 & \\
\hline
\end{tabular}

${ }^{\#}$ values as presented in ref. [1], ${ }^{*}$ assuming substitutional $\mathrm{Br}$ as the only configuration. 

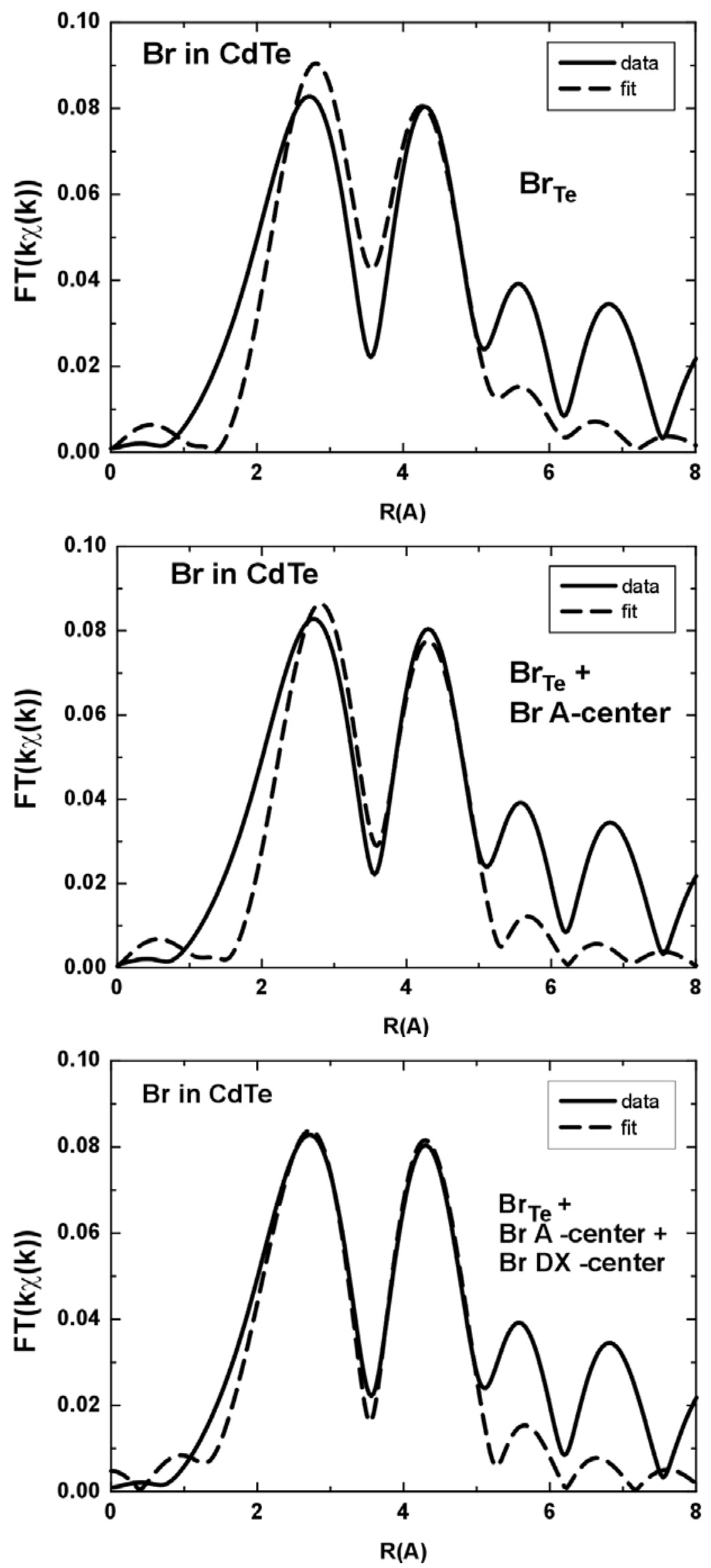

Figure 1. Radial distribution function $\mathrm{FT}(\mathrm{k} \chi(\mathrm{k}))$ of $\mathrm{Br}$ in $\mathrm{CdTe}$ together with a fit assuming substitutional $\mathrm{Br}$ as the only configuration (top), with $60 \%$ of the $\mathrm{Br}$ in the substitutional and $40 \%$ in the A-center configuration (center), and with $\mathrm{Br}$ in 3 configurations, $30 \%$ in the substitutional, $40 \%$ in the A-center and $30 \%$ in the DXcenter configuration (bottom). 


\section{References}

* Corresponding author: Tel. +49-30-8062-2715; Fax. +49-30-8062-2293; E-mail: mahnke@hmi.de

[1] S. Lany et al., Phys. Rev. B 62, R2259 (2000)

[2] V. Ostheimer et al., Phys. Rev. B 68, 235206 (2003)

[3] S. Lany, Elektronische und strukturelle Eigenschaften von Punktdefekten in II-VI Halbleitern, PhD thesis, Universität des Saarlandes, Saarbrücken (Shaker, Aachen 2003) ISBN 3-8322-1302-3.

[4] W. Stadler et al., Phys. Rev. B51, 10619 (1995)

[5] C.H. Park and D.J. Chadi, Phys. Rev. B 52, 11884 (1995)

[6] e.g. S. Lany et al., Physica B302-303, 114 (2001) and ref. therein.

[7] J.J. Rehr, J. Mustre de Leon, S.I. Zabinsky, and R.C. Albers, J. Am. Chem. Soc.113, 5135 (1991)

[8] E.A. Stern, M. Newville, B. Ravel, Y. Yacoby, and D. Haskel, Physica B208\&209, $117(1995)$

[9] M. Newville, P. Livins, Y.Yacoby, J.J. Rehr, and E.A. Stern, Phys. Rev. B47, 14126 (1993)

[10] P. Blaha, K. Schwarz, J.Luitz, WIEN97, A Full Potential Linearized Augmented Plane Wave Package for Calculating Crystal Properties, (Karlheinz Schwarz, TU Wien, Austria, 1999), ISBN 3-9501031-0-4.

[11] M. Bockstedte, A. Kley, J. Neugebauer and M. Scheffler, Comp. Phys. Comm. 107, 187 (1997)

[12] V. Koteski, N. Ivanovic, H. Haas, E. Holub-Krappe, H.-E. Mahnke, NIM B200, 60 (2003)

[13] V. Koteski, H. Haas, E. Holub-Krappe, N. Ivanovic, H.-E. Mahnke, Physica Scripta T115, 369 (2005)

[14] H.-E. Mahnke, H. Haas, E. Holub-Krappe, V. Koteski, N. Novakovic, P. Fochuk, O. Panchuk, Thin Solid Films 480 - 481, 279 (2005) 